\title{
A 54-Year-Old Female With Fever and Ataxia
}

\author{
Marie-Lie Cadieux-Simard MD, Laurence Green MD
}

\begin{abstract}
About the Author
Marie-Li Cadieux-Simard is a 4th year resident in general internal medicine at McGill University. Dr Green is an Associate Professor of Medicine at McGill University and an attending physician at the MUHC. He is the Associate Director for the MUHC Division of General Internal Medicine, and the Department of Medicine Site Director at the Montreal General Hospital. He currently is the Director of the 15 West clinical teaching unit. His interests are in hypertension, pre-operative medicine, tropical medicine and clinical teaching. Correspondence may be directed to marie-lie.cadieux-simard@mail.mcgill.ca.
\end{abstract}

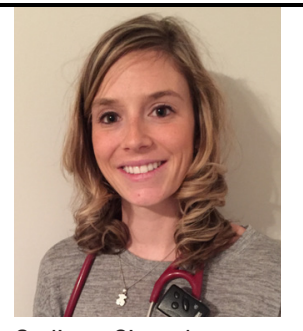

Cadieux-Simard

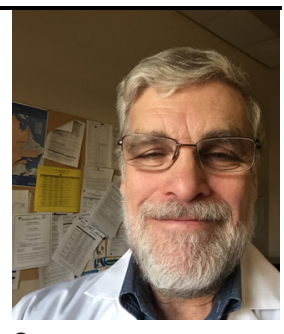

Green

\section{Case Report}

We present the case of a 54-year-old female known for hypertension, alcoholism and occasional cocaine intake. She was brought to the emergency department because of new onset confusion and decreased level of consciousness.

The history was taken from a family member. The patient had increased shortness of breath with a cough that started 48 hours prior to her visit. The night prior to presentation, she was intoxicated with cocaine and alcohol and was then found to have slurred speech, gait disturbance and urinary incontinence. The next morning, she was found obtunded, which prompted her family members to seek medical attention.

In the emergency department, the patient was found to be lethargic and unable to answer question appropriately. Her blood pressure was 140/65, her pulse was 105 and regular, her temperature was $39 \mathrm{oC}$ and her Oxygen saturation was $93 \%$ on room air. There were no signs of respiratory distress.

Given the patient's drowsiness and altered mental status, a computed tomography of her head was done which revealed an age-indeterminate lacunar infarct in the corona radiate. The patient was started on antibiotics to cover for possible bacterial and viral meningitis but these were stopped shortly thereafter because of a normal lumbar puncture.

As part of basic investigations, a chest $\mathrm{x}$-ray was obtained which showed bilateral basal infiltrates. Ceftriaxone and azithromycin were initiated for presumed aspiration or community acquired pneumonia.

Her initial laboratory blood work was significant for thrombocytopenia at $77 \times 10^{9} / \mathrm{L}$, a creatinine of $204 \mu \mathrm{mol} / \mathrm{L}$ and a creatine kinase of $17000 \mathrm{U} / \mathrm{L}$. Her transaminases were three times the upper limit of normal and a toxicology screen was positive for cocaine. Her haemoglobin, white blood cell count $\left(6,2 \times 10^{9} / \mathrm{L}\right)$, and electrolytes were unremarkable. Aggressive fluid resuscitation with isotonic fluids was provided to treat the rhabdomyolysis.

The next day, she was admitted to the internal medicine ward. The admitting diagnosis was delirium secondary to recent intoxication, substance withdrawal, aspiration pneumonia and a possible stroke.

Her mental status improved quickly and a complete neurological assessment was performed. The patient was found to have significant cerebellar findings including gait ataxia, dysarthria, dysmetria and dysdiadochokinesis. Her main complaint at that time was profuse diarrhea. Given the association of gastrointestinal symptoms with pneumonia, we considered the possibility of Legionnaire's disease. A positive urine assay for legionella antigen confirmed the diagnosis. A magnetic resonance imaging was negative but did confirm that the stroke was not acute.

Legionella is one of the leading cause of communityacquired pneumonia and, is also an important pathogen in hospital-acquired pneumonia. It is an important respiratory pathogen, which may have a multisystemic presentation in addition to pneumonia. The classical presentation of Legionnaire's disease includes pneumonia ( $90 \%$ of the cases $)^{1}$ associated with gastrointestinal manifestations, but it has been associated with neurological symptoms in up to $43 \%{ }^{2}$ The most common symptoms are altered mental status and headache. It has also been described in association with liver dysfunction, ${ }^{3}$ hyponatremia and elevated creatine kinase leading to acute renal failure. ${ }^{4}$ Retrospectively, the patient presented with many features that are known to be associated 
with Legionella but the predominance of cerebellar dysfunction as the primary neurologic manifestation is unusual and noted on few case reports. In a computer-based search ${ }^{5}$ including 29 patients with cerebellar dysfunction in the context of legionella, dysarthria and ataxia were the two most common findings. Most of the patients were young otherwise healthy adults. Some had persistent deficits. Computed tomography, magnetic resonance imaging and lumbar puncture are often normal. Treatment is the same as for other Legionellosis cases.

In our case, the antibiotics were tailored to azithromycin alone and the patient's pneumonia improved. Her renal failure, thrombocytopenia and liver dysfunction normalized. Her gait ataxia and dysarthria were still significant at the time of discharge but completely resolved over the following 6 months.

Cigarette smoking and chronic lung disease are known risk factor amongst other for Legionella. There is conflicting data about alcohol and its potential association with the disease and its severity. It would certainly be interesting to better know the risk factor associated with severe disease to identify patients at high risk. Legionnaire's disease is a disease that may present with a variety of symptoms. In our case, the cerebellar symptoms were striking and suggested a primary neurological disease or complication. It is important to consider Legionella infection in patients with pneumonia and neurological findings.

\section{References}

1. Marston BJ, Lipman HB, Breiman RF. Surveillance for Legionnaire's disease: Risk factors for morbidity and mortality. Arch Intern Med 1994; 19: 11301132.

2. Johnson JD, Raff MJ, Arsdall JA. Neurological manifestations of Legionnaire's disease. Medicine 1984; 63: 303-310.

3. Atypical Presentation of Legionnaire's Disease: A Case Report and Review. New York Medical Journal.

4. Johnson DA, Elter HS. Legionnaire's disease with rhabdomyolysis and acute reversible myoglobinuric renal failure. South Med J 1984; 77: 777-779.

5. Southern Medical Journal. Cerebellar Involvement in Legionellosis. Shelburne, Samuel A. MD, Kielhofner, Marcia A. MD, Tiwari, Pinky S. MD South Med J. 2004;97(1). 